\title{
Implicación del patrón radiológico en el pronóstico de los tumores oligodendrogliales: correlación con el perfil genético
}

\author{
Marta Brell, Sara Castañer, Teresa Pujol-Farré, Susana Boluda, Teresa Ribalta, Jordi Bruna, María Martínez, \\ Eugenia Verger, Salvador Villà, Nuria Viñolas, Miguel Gil, Francesc Graus, Avelina Tortosa-Moreno
}

Introducción. La codeleción 1p19q (LOH1p19q) confiere a los tumores oligodendrogliales quimiosensibilidad y un mejor pronóstico en relación con otros gliomas. La investigación dirigida a identificar características radiológicas asociadas a LOH1p19q ha despertado gran interés en los últimos años.

Objetivos. Confirmar la existencia de heterogeneidad regional de los parámetros moleculares en los gliomas oligodendrogliales, valorar la asociación entre el perfil genético y determinadas características radiológicas y clínicas, y analizar el valor pronóstico de éstas.

Pacientes y métodos. Se incluyeron 54 pacientes tratados según un protocolo preestablecido común. Se valoraron las secuencias $T_{1}$, con/sin gadolinio, y $T_{2}$ de la resonancia magnética preoperatoria a ciegas de la información molecular y clínica. El análisis de LOH se efectuó sobre muestras pareadas de ADN tumoral y genómico.

Resultados. La presencia de LOH1p se halló fuertemente asociada a LOH19q ( $p<0,0001)$. LOH1p19q resultó más frecuente en los tumores situados en el lóbulo frontal (odds ratio, OR = 5,38; intervalo de confianza del 95\%, IC 95\% = 1,51-19,13; $p=0,007)$ y sin necrosis radiológica ( $O R=0,17 ;$ IC $95 \%=0,03-0,80 ; p=0,02)$. La localización frontal (riesgo relativo, $\mathrm{RR}=4,499 ;$ IC 95\% = 1,027-193,708; $p=0,046)$, la necrosis radiológica ( $\mathrm{RR}=0,213 ;$ IC 95\% =0,065-0,700; $p=0,011)$ y el grado de resección ( $R R=9,231 ;$ IC $95 \%=1,737-49,050 ; p=0,009)$ resultaron factores pronósticos independientes de supervivencia global.

Conclusiones. En los tumores oligodendrogliales, además del análisis histológico y el estudio genético-molecular, la valoración de determinadas características radiológicas puede resultar de gran utilidad para definir subgrupos de pacientes con pronóstico y respuesta al tratamiento similares. Los esfuerzos deben dirigirse, por tanto, hacia la utilización combinada de todos los recursos disponibles en cada centro.

Palabras clave. LOH. Oligoastrocitoma. Oligodendroglioma. Pronóstico. Resonancia magnética. Supervivencia.

\section{Introducción}

Los tumores oligodendrogliales, tanto puros como mixtos, presentan mejor pronóstico que los astrocitarios [1]. Un alto porcentaje de los tumores oligodendrogliales muestra pérdida de heterocigosidad de $1 p 19 q$ (LOH1p19q). La presencia de ésta, y particularmente de $\mathrm{LOH} 1 p$, les confiere mayor quimiosensibilidad y mejor pronóstico en relación con otros gliomas [2]; sin embargo, la clasificación histopatológica actual sigue basándose en criterios morfológicos, por lo que resulta compleja en ocasiones, además de insuficiente para predecir la respuesta al tratamiento o el pronóstico de los pacientes [3]. La investigación dirigida a la identificación de características radiológicas asociadas a $\mathrm{LOH} 1 p 19 q$ y que, por tanto, permitan predecir el comportamiento biológi- co del tumor, ha despertado un gran interés en los últimos años [4,5-10]. El fundamento científico sobre el que se sustenta esta relación genético-radiológica es el hecho de que la codeleción $1 p 19 q$-evento precoz en la génesis de los gliomas oligodendrogliales- podría traducir una menor capacidad invasiva e infiltrativa de aquellos tumores que la poseen, y condicionar así su aspecto radiológico, localización y extensión en el cerebro [11]. No obstante, la información publicada hasta la fecha es limitada y controvertida porque la mayoría de estudios son retrospectivos, usan clasificaciones de la Organización Mundial de la Salud (OMS) previas a la vigente o incluyen esquemas terapéuticos heterogéneos, por lo que el análisis de factores pronósticos resulta poco fidedigno.

Sobre la hipótesis de que determinadas características radiológicas en pacientes con tumores oli-
Servicio de Radiología (S. Castañer) Servicio de Anatomía Patológica (S. Boluda); Servicio de Neurología (J. Bruna); Hospital Universitari de Bellvitge. Servicio de Oncología Médica; Institut Català d'Oncologia; IDIBELL (M. Gil). Departamento de Enfermería Fundamental; IDIBELL; Universitat de Barcelona (A. TortosaMoreno); L'Hospitalet de Llobregat, Barcelona. Servicio de Radiología (T. Pujol-Farré); Servicio de Anatomía Patológica (T. Ribalta); Servicio de Oncología Radioterápica (E. Verger); Servicio de Oncología Médica (N. Viñolas); Servicio de Neurología (F. Graus); Hospital Clínic; Barcelona. Servicio de Oncología Médica; Hospital del Mar (M. Martínez); Barcelona. Servicio de Oncología Radioterápica; Institut Català d'Oncologia; Hospital Germans Trias i Pujol (S. Villà); Badalona, Barcelona. Servicio de Neurocirugía; Hospital Universitario Son Dureta (M. Brell); Palma de Mallorca, España.

Correspondencia:

Dra. Avelina Tortosa Moreno. Departamento de Enfermería Fundamental. IDIBELL. Campus de Bellvitge. Universitat de Barcelona. Feixa Llarga, s/n. Pavelló Central, 3. a planta. E-08907 L'Hospitalet de Llobregat (Barcelona).

Fax:

+34934024216

E-mail:

atortosa@ub.edu

Financiación:

Financiado parcialmente por la beca FIS 08-1085 del Ministerio de Sanidad y Consumo y por la beca Fundación Mutua Madrileña 2007.

Agradecimientos:

Al Dr. A. Castells, por su soporte estadístico.

Aceptado tras revisión externa: 05.10.10.

Cómo citar este artículo:

Brell M, Castañer S, Pujol-Farré T, Boluda S, Ribalta T, Bruna J, et al. 


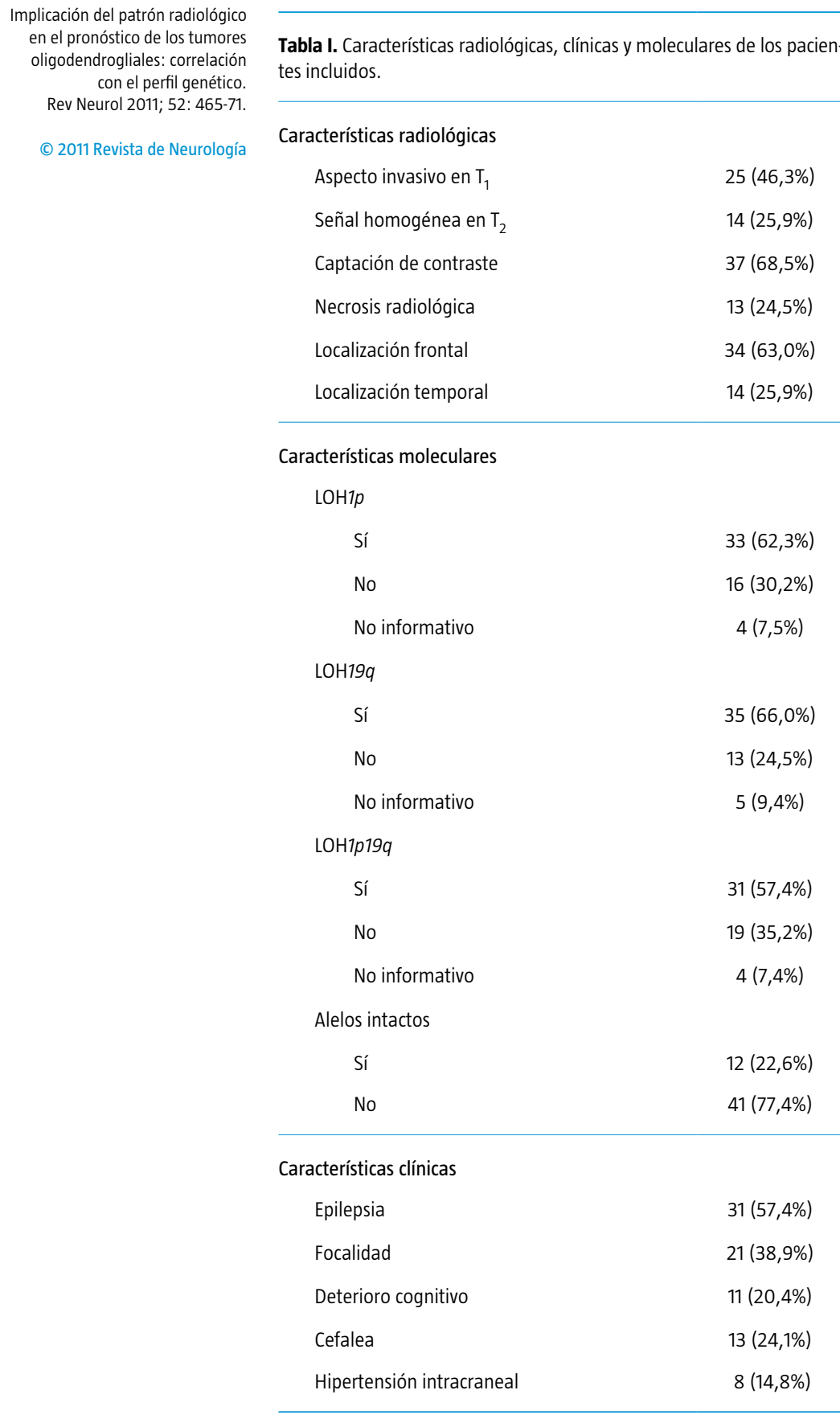

godendrogliales puede definir subgrupos con pronóstico similar, los objetivos del presente trabajo fueron los siguientes: confirmar la existencia de heterogeneidad regional de los parámetros moleculares en una serie de pacientes con diagnóstico consensuado de tumor oligodendroglial -tratados de acuerdo a un protocolo diagnóstico y terapéutico común-, valorar la asociación entre el perfil genético y determinadas características radiológicas y clínicas y analizar el valor pronóstico en términos de supervivencia global y supervivencia libre de progresión de las variables radiológicas, clínicas y moleculares estudiadas.

\section{Pacientes y métodos}

Se incluyeron 54 pacientes con oligodendroglioma u oligoastrocitoma de grado II y III de la OMS. Todos los tumores fueron revisados y clasificados por dos neuropatólogos independientes, siguiendo la última actualización de la OMS [2]. Las muestras se recogieron durante la cirugía, se dejaron parafinadas para el estudio histológico y, en los casos con excedente, congeladas y almacenadas a $-80^{\circ} \mathrm{C}$. Se consideraron tumores mixtos aquéllos con componente astrocitario y oligodendroglial en áreas geográficamente distintas y con un mínimo de componente oligodendroglial del $25 \%$. El estudio fue aprobado por el Comité de Ética Asistencial y todos los pacientes dieron su consentimiento informado para participar.

Se aplicó un protocolo preestablecido común de diagnóstico y tratamiento que incluía cirugía, seguida de radio y quimioterapia para los gliomas de grado III, y de radioterapia para los de grado II resecados parcialmente o en recidiva/progresión. Los pacientes con deterioro del estado general o situación funcional precaria no recibieron tratamiento complementario. La radioterapia consistió en irradiación focal sobre el lecho tumoral más $2-3 \mathrm{~cm}$ de margen, con una dosis total de 50-54 Gy en los de grado II y de 60 Gy en los de grado III, en fracciones de 2 Gy/ día. La quimioterapia consistió en carmustina (200 $\mathrm{mg} / \mathrm{m}^{2}$ cada 8 semanas $\times 6$ ciclos), o PCV (procarbacina, $60 \mathrm{mg} / \mathrm{m}^{2}$ los días $8-21$; lomustina, $110 \mathrm{mg} / \mathrm{m}^{2}$ el día 1 , y vincristina, $1,4 \mathrm{mg} / \mathrm{m}^{2}$ los días 8 y 29 cada 6 semanas $\times 6$ ciclos). Quedaron excluidos de la quimioterapia aquellos pacientes que presentaron progresión durante la radioterapia. La respuesta al tratamiento se evaluó mediante resonancia magnética (RM) según los criterios de Macdonald et al [12].

$\mathrm{El}$ análisis radiológico fue realizado por dos neurorradiólogos independientes a ciegas de la información molecular y clínica, y de acuerdo a la metodología publicada previamente [7,9]. Se valoraron en las secuencias $\mathrm{T}_{1}$, con/sin gadolinio, y $\mathrm{T}_{2}$ de la $\mathrm{RM}$ preoperatoria el aspecto invasivo en $\mathrm{T}_{1}$ (límites mal definidos frente a límites bien definidos), la señal homogénea frente a heterogénea en $\mathrm{T}_{2}$, la presencia frente a la ausencia de captación de contraste, la presencia frente a la ausencia de necrosis ra- 
diológica y la localización. Se consideró como localización aquel lóbulo en el que residía la mayor parte de la masa tumoral.

El ADN tumoral se obtuvo de las muestras parafinadas o congeladas de acuerdo con protocolos previos [13], y el ADN genómico de linfocitos de sangre periférica se consiguió con el kit comercial QIAamp ${ }^{\circledR} 96$ DNA Blood Kit (Qiagen, Alemania). Se estudió la $\mathrm{LOH}$ en muestras pareadas de ADN tumoral y genómico utilizando marcadores de microsatélite en 1p36 (D1S2734, D1S199, D1S508) y 19q13 (D19S219, D19S112, D19S412, D19S596). Las condiciones de amplificación y la secuencia de los cebadores se obtuvieron de la base de datos Genome Database (www.ncbi.nlm.nih.gov/sites/genome). Los productos de la reacción en cadena de la polimerasa se examinaron con el analizador Applied Biosystems 3730 DNA Analyzer, y el patrón de los alelos, con el software GeneMapper 3.0 (Applied Biosystems). Las variables clínicas recogidas fueron sintomatología de presentación (epilepsia, focalidad, deterioro cognitivo, cefalea e hipertensión intracraneal) y el tipo de cirugía (biopsia, resección parcial o completa) [14].

El análisis estadístico de la asociación entre las características radiológicas/clínicas y moleculares se efectuó mediante la prueba de chi al cuadrado y el test exacto de Fisher. La localización se dicotomizó en frontal frente a no frontal de acuerdo con estudios previos [5,9]. La supervivencia global se calculó desde la fecha de la intervención quirúrgica hasta el momento del fallecimiento, y la supervivencia libre de progresión, desde la cirugía hasta la recidiva/progresión. Las curvas de supervivencia se elaboraron según el método de Kaplan-Meier y se compararon mediante el test de log-rank. El análisis de factores pronósticos se realizó sobre el subgrupo de pacientes con gliomas anaplásicos. Las variables con nivel de significación $p \leq 0,1$ en el análisis univariante se incluyeron en el multivariante según el modelo de riesgos proporcionales de Cox.

Se empleó el programa estadístico SPSS v. 16.0 y un nivel de significación de $p=0,05$.

\section{Resultados}

De los 54 pacientes incluidos en el estudio, $16(29,6 \%)$ fueron de grado II (10 con oligodendroglioma y 6 con oligoastrocitoma) y 38 (70,4\%) de grado III (18 con oligodendroglioma y 20 con oligoastrocitoma). Veintiséis $(48,1 \%)$ eran hombres; la mediana de edad fue de 47 años (rango: 21-78 años). Se dispuso de seguimiento clínico en todos los casos con una me- diana de 76,1 meses (rango: 20,17-168,5 meses). Las características clínicas, radiológicas y moleculares se muestran en la tabla I. En 16 (29,6\%) pacientes se realizó una resección completa, mientras que en 38 $(70,4 \%)$ sólo parcial $(n=31)$ o biopsia $(n=7)$. La localización del tumor no condicionó el grado de resección $(p=0,56)$. Tres pacientes $(7,8 \%)$ con gliomas anaplásicos no recibieron radioterapia -dos por haberla recibido previamente y uno por mal estado general- y seis $(15,7 \%)$ no recibieron quimioterapia por antecedentes patológicos que lo contraindicaban en un caso y por situación funcional precaria en el resto. De los pacientes con tumores de grado II, siete $(43,7 \%)$ recibieron radioterapia, cuatro tras resecciones incompletas y tres por existir recidiva/progresión de gliomas previos. Un paciente con oligoastrocitoma de grado II recibió quimioterapia durante la progresión.

Se dispuso de material para el análisis molecular en 53 casos $(98,1 \%)$. Se halló una fuerte asociación entre LOH1 $1 p$ y LOH $19 q$, de modo que $31(96,8 \%)$ de los 32 tumores con LOH1 $1 p$ presentaban también LOH19q, y a la inversa, 31 (88,5\%) de los 35 tumores con LOH19q mostraron $\mathrm{LOH} 1 p$ concomitante $(p<0,0001)$. En el subanálisis por tipo histológico, se halló $\mathrm{LOH} 1 p$ en el $76 \%$ de los oligodendrogliomas y en el $58,3 \%$ de los tumores mixtos $(p=0,18)$; LOH $19 q$ en el $79,1 \%$ de los oligodendrogliomas y en el $66,6 \%$ de los oligoastrocitomas $(p=0,33)$ y LOH $1 p 19 q$ en el $68 \%$ de los tumores puros y en el $58,3 \%$ de los mixtos $(p=0,48)$. En relación con el grado, se detectó LOH1p en el 73,3\% de los tumores de grado II y en el $64,7 \%$ de los de grado III ( $p=$ $0,74)$, $\mathrm{LOH} 19 q$ en el $68,7 \%$ de los tumores de bajo grado y en el $75 \%$ de los anaplásicos $(p=0,73)$ y LOH $1 p 19 q$ en el $62,5 \%$ de los tumores de bajo grado frente al 63,6\% de los anaplásicos $(p=0,93)$.

El análisis de correlación con las características radiológicas demostró una asociación estadísticamente significativa entre localización frontal y $\mathrm{LOH} 1 p$ (odds ratio, $\mathrm{OR}=6,19$; intervalo de confianza del 95\%, IC 95\% = 1,66-22,68; $p=0,004), \mathrm{LOH} 19 q$ $(\mathrm{OR}=7,59 ;$ IC $95 \%=1,84-31,34 ; p=0,006) \mathrm{y}$ LOH $1 p 19 q(\mathrm{OR}=5,38$; IC 95\% = 1,51-19,13; $p=$ $0,007)$, así como entre ausencia de necrosis radiológica y codeleción $1 p 19 q(\mathrm{OR}=0,17$; IC $95 \%=0,03$ $0,80, p=0,02$ ). Esta asociación se mantuvo en el subanálisis realizado sobre los tumores mixtos -localización frontal y LOH1 $p(p=0,006)$, localización frontal y LOH $19 q(p=0,001)$, localización frontal y LOH $1 p 19 q(p=0,006)-\mathrm{y}$ anaplásicos -localización frontal y LOH $1 p(p=0,02)$, localización frontal y LOH $19 q(\mathrm{p}=0,002)$, localización frontal y LOH1p19q $(p=0,02)-$, en los que también se observó asocia- 
Figura 1. Curva de supervivencia según el método de Kaplan-Meier para localización.

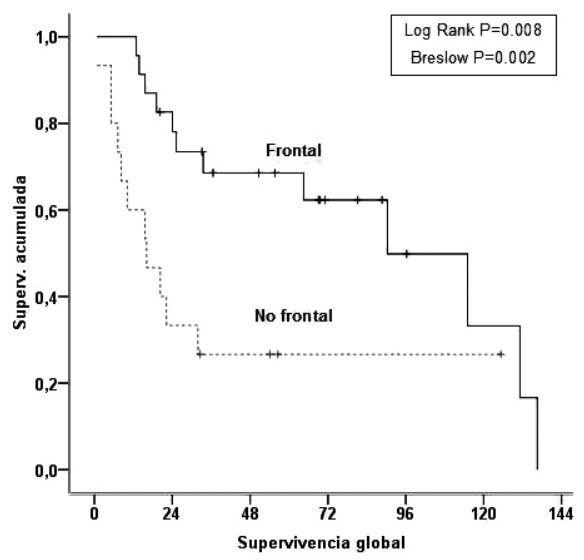

Figura 3. Supervivencia global según el método de Kaplan-Meier para LOH1p19q.

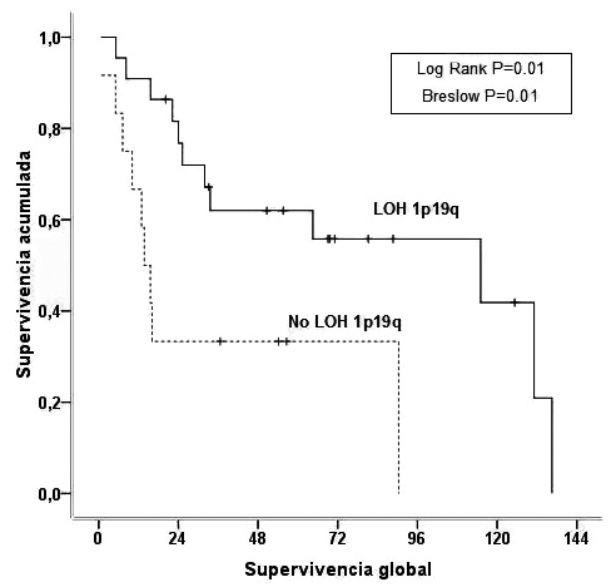

ción significativa entre ausencia de necrosis radiológica y $\mathrm{LOH} 1 p 19 q(p=0,04)$. El resto de los aspectos radiológicos analizados no demostró asociación significativa con las características moleculares. De las variables clínicas, sólo la focalidad resultó significativamente menor en los pacientes cuyos tumores presentaban LOH1 $p(p=0,048)$.

La mediana de supervivencia global resultó significativamente menor en los pacientes con glioma anaplásico, 64,5 meses (rango: 3,47-125,5 meses), no alcanzada en los pacientes con glioma de grado II
Figura 2. Curva de supervivencia según el método de Kaplan-Meier para necrosis radiológica.

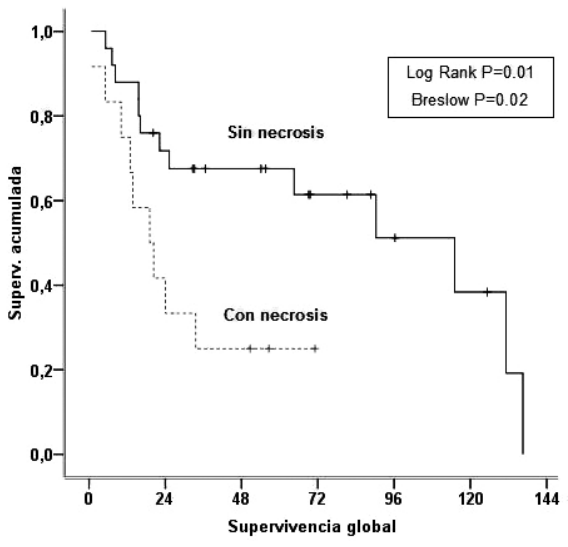

Figura 4. Supervivencia libre de progresión según el método de KaplanMeier para LOH1p19q.

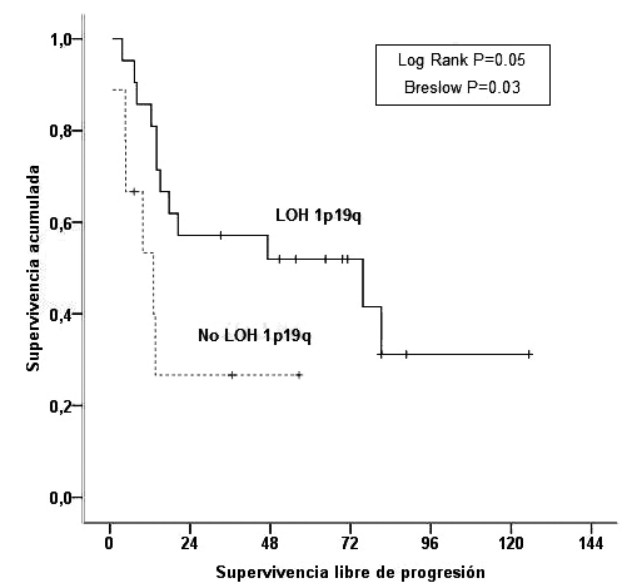

$(p=0,002)$. La mediana de supervivencia libre de progresión fue de 20,3 meses (rango: 0-54,9 meses) para los tumores anaplásicos y de 54,7 meses (rango: 38,2-71,3 meses) para los de bajo grado. En el análisis univariante la necrosis radiológica y la localización mostraron un impacto significativo en la supervivencia global (Figs. 1 y 2). El grado de resección sólo mostró una tendencia hacia la significación estadística $(p=0,104)$, mientras que la histología pura frente a mixta no resultó un factor pronóstico. Por lo que respecta a las características molecula- 
Tabla II. Análisis multivariante de factores pronósticos en los gliomas anaplásicos $(n=38)$.

\begin{tabular}{lcccc}
\hline & \multicolumn{2}{c}{ Supervivencia global } & \multicolumn{2}{c}{ Supervivencia libre de progresión } \\
\cline { 2 - 5 } & RR (IC 95\%) & $p$ & RR (IC 95\%) & $p$ \\
\hline Localización frontal & $4,499(1,027-19,708)$ & $0,046^{\text {a }}$ & $1,174(0,469-2,939)$ & 0,732 \\
\hline Presencia de necrosis & $0,213(0,065-0,700)$ & $0,011^{\text {a }}$ & $0,868(0,476-1,585)$ & 0,645 \\
\hline Resección completa & $9,231(1,737-49,050)$ & $0,009^{\text {a }}$ & $13,572(1,461-126,069)$ & $0,022^{\text {a }}$ \\
\hline Presencia de LOH1p19q & $1,755(0,405-7,605)$ & 0,452 & $1,174(0,469-2,939)$ & 0,732 \\
\hline
\end{tabular}

RR: riesgo relativo; IC 95\%: intervalo de confianza del 95\%. a Resultados estadísticamente significativos.

res, $\mathrm{LOH} 1 p$, LOH $19 q$ y LOH $1 p 19 q$ resultaron factores pronósticos de supervivencia global y libre de progresión (Figs. 3 y 4). En el análisis multivariante la localización frontal, la necrosis radiológica y el grado de resección permanecieron como factores pronósticos independientes de supervivencia global, mientras que sólo el grado de resección resultó un factor pronóstico independiente de supervivencia libre de progresión (Tabla II).

En el subanálisis realizado sobre los pacientes con oligoastrocitomas, la localización (hazard ratio, $\mathrm{HR}=3,834 ;$ IC 95\% = 1,053-13,949; $p=0,041) \mathrm{y}$ la necrosis radiológica $(\mathrm{HR}=0,125$; IC $95 \%=0,034$ $0,457 ; p=0,001)$ también mostraron impacto pronóstico en el análisis univariante, junto con las características moleculares: $\mathrm{LOH} 1 p(\mathrm{HR}=5,224$; IC 95\% = 1,314-20,765; $p=0,018), \mathrm{LOH} 19 q(\mathrm{HR}=$ 5,329; IC 95\% = 1,379-20,592; $p=0,015)$, LOH $1 p 19 q$ $(\mathrm{HR}=5,224 ; \mathrm{IC} 95 \%=1,314-20,765 ; p=0,018)$. La necrosis radiológica fue la única variable con influencia pronóstica en el análisis multivariado $(\mathrm{HR}=0,046$; IC 95\% = 0,006-0,332; $p=0,002)$.

\section{Discusión}

Los resultados del presente estudio demuestran que los oligodendrogliomas puros o mixtos situados en el lóbulo frontal presentan con mayor frecuencia LOH1p19q, mientras que aquéllos con alelos intactos asientan fundamentalmente en el lóbulo temporal, y corroboran, por lo tanto, la existencia de heterogeneidad molecular dependiente de la región en los tumores oligodendrogliales $[5,6,8,9,15,16]$. El fundamento biológico de esta asociación genéticoradiológica continúa siendo desconocido en la actualidad, y se especula con dos hipótesis. En primer lugar, se ha relacionado con aspectos del medio extracelular [17], de forma que factores de crecimiento secretados por áreas concretas del cerebro facilitarían el desarrollo específico de determinadas células neoplásicas; por ejemplo, aquéllas con LOH $1 p 19 q$ $[1,9]$. La segunda posibilidad es que la diferenciación de la extirpe oligodendroglial se produzca de manera muy precoz durante el desarrollo embrionario, de manera que se originen distintas subpoblaciones de oligodendrocitos que adoptarán diferentes vías de migración colonizando de manera dirigida áreas cerebrales específicas [18-20].

Asimismo, en nuestro estudio la localización frontal y la ausencia de necrosis radiológica se asociaron de forma independiente a una mayor supervivencia. Algunos trabajos previos describen menor quimiosensibilidad de los oligodendrogliomas anaplásicos localizados en el lóbulo temporal/ínsula/ diencéfalo, sin que ello se traduzca en diferencias significativas en la supervivencia de los pacientes [9]. Existe la posibilidad de que el impacto pronóstico de las variables radiológicas se deba en realidad a su asociación con las alteraciones moleculares [21], y sea simplemente una expresión subrogada de éstas. De hecho, en nuestra serie la localización frontal y la necrosis radiológica se asociaron de manera significativa a LOH1p19q. Por ello, tanto los factores moleculares como los radiológicos se incluyeron en el análisis multivariante, y permanecieron la localización y la necrosis como factores pronósticos independientes. El número limitado de pacientes obliga, no obstante, a considerar estos resultados con precaución y justifica probablemente que las alteraciones cromosómicas no hayan permanecido como factores independientes en el análisis multivariado [22]. De todas formas, existe también la posibilidad de que durante el desarrollo los 
tumores experimenten modificaciones epigenéticas con impacto en su evolución [23].

En lo referente al grado de resección, los resultados de nuestra serie confirman que la resección completa es factor pronóstico independiente de supervivencia global y libre de progresión, tal como se había postulado anteriormente [24,25]. Aunque la topografía tumoral puede condicionar las opciones quirúrgicas, y por lo tanto de manera indirecta el pronóstico, no hallamos en nuestra serie una asociación significativa entre la localización y el grado de resección.

Respecto a la histología, no encontramos diferencias significativas en la supervivencia de los pacientes con oligodendrogliomas anaplásicos en relación con aquéllos con oligoastrocitomas anaplásicos, ni tampoco en el perfil molecular en función de la histología pura o mixta, con lo que se corroboran los resultados de series previas [24-27]. Aunque existe cierta controversia, parece ser el genotipo subyacente lo que verdaderamente condiciona el pronóstico de los pacientes con gliomas mixtos y no el subtipo histológico $[15,28]$.

En conclusión, el objetivo de clasificar los gliomas es definir subgrupos de pacientes con un pronóstico y respuesta al tratamiento similares. Para ello, además del análisis histológico, el estudio genético proporciona información muy valiosa. Nuestros resultados confirman que las deleciones de $1 p, 19 q \mathrm{y}$ $1 p 19 q$ son más frecuentes en los tumores oligodendrogliales que asientan en el lóbulo frontal y carecen de necrosis radiológica. Además, estas características radiológicas poseen per se influencia pronóstica; sin embargo, la asociación genético-radiológica adolece de algunas excepciones, por lo que resulta improbable que pueda llegar a predecirse el estado de $1 p 19 q$ tomando como base exclusivamente aspectos radiológicos [11]. Los esfuerzos deben dirigirse, por tanto, hacia la utilización combinada de todos los recursos disponibles en cada centro (radiología, inmunohistoquímica, aspectos clínicos y estudio genético) $[6,29,30]$. Se requieren series mayores para validar estos resultados preliminares.

\section{Bibliografía}

1. Huang L, Jiang T, Yuan F, Li GL, Cui Y, Liu EZ, et al. Correlation of chromosomes $1 \mathrm{p}$ and $19 \mathrm{q}$ status and expressions of O6-methylguanine DNA methyltransferase (MGMT), p53 and Ki-67 in diffuse gliomas of World Health Organization (WHO) grades II and III: a clinicopathological study. Neuropathol Appl Neurobiol 2009; 35: 367-79.

2. Louis DN, Ohgaki H, Wiestler OD, Cavenee WK. The WHO classification of tumors of the central nervous system. 4 ed. Lyon: IARC; 2007.

3. Blesa D, Mollejo M, Ruano Y, De Lope AR, Fiano C, Ribalta
T, et al. Novel genomic alterations and mechanisms associated with tumor progression in oligodendroglioma and mixed oligoastrocytoma. J Neuropathol Exp Neurol 2009; 68: 274-85.

4. Walker C, Du Plessis DG, Joyce KA, Fildes D, Gee A, Haylock $\mathrm{B}$, et al. Molecular pathology and clinical characteristics of oligodendroglial neoplasms. Ann Neurol 2005; 57: 855-65.

5. Laigle-Donadey F, Martin-Duverneuil N, Lejeune J, Crinière E, Capelle L, Duffau H, et al. Correlations between molecular profile and radiologic pattern in oligodendroglial tumors. Neurology 2004; 63: 2360-2.

6. McDonald JM, See SJ, Tremont IW, Colman H, Gilbert MR, Groves $\mathrm{M}$, et al. The prognostic impact of histology and $1 \mathrm{p} / 19 \mathrm{q}$ status in anaplastic oligodendroglial tumors. Cancer 2005; 104: 1468-77.

7. Megyesi JF, Kachur E, Lee DH, Zlatescu MC, Betensky RA, Forsyth PA, et al. Imaging correlates of molecular signatures in oligodendrogliomas. Clin Cancer Res 2004; 10: 4303-6.

8. Mueller W, Hartmann C, Hoffmann A, Lanksch W, Kiwith J, Tonn J, et al. Genetic signature of oligoastrocytomas correlates with tumor location and denotes distinct molecular subsets. Am J Pathol 2002; 161: 313-9.

9. Zlatescu MC, Tehrani Yazdi A, Sasaki H, Megyesi JF, Betensky RA, Louis DN, et al. Tumor location and growth patter correlate with genetic signature in oligodendroglial neoplasms. Cancer Res 2001; 61: 6713-5.

10. Whitmore RG, Krejza J, Kapoor GS, Huse J, Woo JH, Bloom $\mathrm{S}$, et al. Prediction of oligodendroglial tumor subtype and grade using perfusion weighted magnetic resonance imaging. J Neurosurg 2007; 107: 600-9.

11. Jenkinson MD, Du Plessis DG, Smith TS, Joyce KA, Warnke $\mathrm{PC}$, Walker C. Histological growth patterns and genotype in oligodendroglial tumours: correlation with MRI features. Brain 2006; 129: 1884-91.

12. Macdonald DR, Cascino TL, Schold SC Jr, Cairncross JG. Response criteria for phase II studies of supratentorial malignant glioma. J Clin Oncol 1990; 8: 1277-80.

13. Brell M, Tortosa A, Verger E, Gil JM, Viñolas N, Villà $S$, et al. Prognostic significance of $\mathrm{O}_{6}$-methilguanine. DNA methyltransferase determined by promoter methylation and immunohistochemical expression in anaplastic gliomas. Clin Cancer Res 2005; 11: 5167-71.

14. Van den Bent MJ, Looijenga LHJ, Langenberg K, Dinjens W, Graveland W, Uytdewilligen L, et al. Chromosomal anomalies in oligodendroglial tumors are correlated with clinical features. Cancer 2003; 97: 1276-84.

15. Huang L, Jiang T, Yuan F, Li GL, Liu EZ, Wang ZC. Correlations between molecular profile and tumor location in Chinese patients with oligodendroglial tumors. Clin Neurol Neurosurg 2008; 110: 1020-4.

16. Kouwenhoven MC, Gorlia T, Kros JM, Ibdaih A, Brandes AA, Bromberg JE, et al. Molecular analysis of anaplastic oligodendroglial tumors in a prospective randomized study: a report from EORTC study 26951. Neuro Oncol 2009; 11: 737-46.

17. Mukasa A, Ueki K, Matsumoto S, Tsutsumi S, Nishikawa R, Fujimaki T, et al. Distinction in gene expression profiles of oligodendrogliomas with and without allelic loss of $1 \mathrm{p}$. Oncogene 2002; 21: 3961-8.

18. Cairncross JG, Ueki K, Zlatescu MC, Lisle DK, Finkelstein DM, Hammond RR, et al. Specific genetic predictors of chemotherapeutic response and survival in patients with anaplastic oligodendrogliomas. J Natl Cancer Inst 1998; 90: 1473-9.

19. Cairncross JG. Imaging molecular signatures in oligodendroglioma. Cancer Res 2004; 10: 7109-11.

20. López JI, Pomposo-Gaztelu I. Patología quirúrgica de la epilepsia. Rev Neurol 2010; 50: 616-22.

21. Kim SH, Kim H, Kim TS. Clinical, histological, and immunohistochemical features predicting $1 \mathrm{p} / 19 \mathrm{q}$ loss of heterozygosity in oligodendroglial tumors. Acta Neuropathol 2005; 110: 27-38.

22. Weller M, Berger H, Hartmann C, Schramm J, Westphal M, Simon M, et al. Combined 1p/19q loss in oligodendroglial tumors: predictive or prognostic biomarker? Clin Cancer Res 2007; $13: 6933-7$. 
23. Campbell BA, Horsman DE, Maguire J, Young S, Curman D, Ma R, et al. Chromosomal alterations in oligodendroglial tumours over multiple surgeries: is tumour progression associated with change in 1p/19q status? J Neurooncol 2008; 89: 37-45.

24. Park CK, Lee SH, Han JH, Kim CY, Kim DW, Paek SH, et al. Recursive partitioning analysis of prognostic factors in $\mathrm{WHO}$ grade III glioma patients treated with radiotherapy or radiotherapy plus chemotherapy. BMC Cancer 2009; 9: 450.

25. Sanai N, Berger MS. Glioma extent of resection and its impact on patient outcome. Neurosurgery 2008; 62: 753-64.

26. Kanamori M, Kumabe T, Sonoda Y, Nishino Y, Watanabe M, Tominaga T. Predictive factors for overall and progressionfree survival, and dissemination in oligodendroglial tumors. J Neurooncol 2009; 93: 219-28.
27. DeAngelis LM. Anaplastic glioma: how to prognosticate outcome and choose a treatment strategy. J Clin Oncol 2009; 27:5861-2.

28. Vanden BentMJ,ReniM,GattaG, VechtC.Oligodendroglioma. Crit Rev Oncol Hematol 2008; 66: 262-72.

29. Kapoor GS, Gocke TA, Chawla S, Whitmore RG, Nabavizadeh A, Krejza J, et al. Magnetic resonance perfusion-weighted imaging defines angiogenic subtypes of oligodendroglioma according to $1 \mathrm{p} 19 \mathrm{q}$ and EGFR status. J Neurooncol 2009; 92: 373-86.

30. Kim JW, Park CK, Park SH, Kim YH, Han JH, Kim CY, et al. Relationship between radiological characteristics and combined $1 \mathrm{p}$ and $19 \mathrm{q}$ deletion in World Health Organization grade III oligodendroglial tumours. J Neurol Neurosurg Psychiatry 2010; Jun 28. [Epub ahead of print].

Implication of radiological pattern in the prognosis of oligodendroglial tumors: correlation with genetic profile

Introduction. 1p19q loss of heterozygosity (LOH1p19q) in oligodendroglial tumors has shown to be prognostic of prolonged survival and predictive of therapeutic responsiveness. During the last years, research is actively being directed to the discovery of radiological characteristics related to LOH1p19q.

Aims. To confirm the existence of molecular heterogeneity in oligodendroglial tumors in relation to their anatomic distribution, and to evaluate the correlation between molecular profile and other radiological and clinical characteristics and their prognostic impact.

Patients and methods. Fifty-four patients with oligodendroglial tumors managed according to a previously established protocol were included. Preoperative $S E T_{1}, T_{1}$ post-gadolinium and $T_{2}$ magnetic resonance images were reviewed by two independent neuroradiologists, blinded to clinical and molecular information. LOH analysis was assessed from paired tumor-blood DNA acid samples.

Results. LOH $7 p$ was highly associated with LOH19q ( $p<0.0001$ ), LOH7p (odds ratio, OR $=6.19 ; 95 \%$ confidence interval, $95 \% \mathrm{Cl}=1.66-22.68 ; p=0.004)$, LOH19q (OR $=7.59 ; 95 \% \mathrm{Cl}=1.84-31.34 ; p=0.006)$ and LOH1p19q (OR $=5.38 ; 95 \%$ $\mathrm{Cl}=1.51-19.13 ; p=0.007$ ) were found to be more frequent in tumors located in the frontal lobe. Frontal location (hazard ratio, $\mathrm{HR}=4.499 ; 95 \% \mathrm{Cl}=1.027-193.708 ; p=0.046)$, ring enhancement $(\mathrm{HR}=0.213 ; 95 \% \mathrm{Cl}=0.065-0.700 ; p=0.011)$ and extent of resection ( $\mathrm{HR}=9.231 ; 95 \% \mathrm{Cl}=1.737-49.050 ; p=0.009$ ) resulted independent prognostic factors for overall survival in the multivariate analysis.

Conclusions. Glioma classification aims to better define patients prognosis. Besides histological and immunohistochemical analyses, molecular information has become of great importance. Our results indicate that the evaluation of some MR features may also be useful. Efforts must be directed toward the use of every available resource at each institution.

Key words. LOH. Magnetic resonance imaging. Oligoastrocytoma. Oligodendroglioma. Prognosis. Survival. 